\title{
Cytotoxic T Lymphocyte-Associated Antigen 4 Gene Polymorphisms and Autoimmune Thyroid Diseases: An Updated Systematic Review and Cumulative Meta-Analysis
}

\author{
Hai-Feng Hou, ${ }^{1}$ Xu Jin, ${ }^{2}$ Tao Sun, ${ }^{1}$ Cheng Li, ${ }^{3}$ Bao-Fa Jiang, ${ }^{4}$ and Qun-Wei Li ${ }^{1}$ \\ ${ }^{1}$ School of Public Health, Taishan Medical University, Taian 271000, China \\ ${ }^{2}$ School of Basic Medical Sciences, Taishan Medical University, Taian 271000, China \\ ${ }^{3}$ Ruijin Hospital, Shanghai Jiao Tong University, Shanghai 200025, China \\ ${ }^{4}$ School of Public Health, Shandong University, Jinan 250012, China \\ Correspondence should be addressed to Qun-Wei Li; tougaolxb@163.com
}

Received 16 January 2015; Accepted 17 February 2015

Academic Editor: Maria L. Dufau

Copyright (C) 2015 Hai-Feng Hou et al. This is an open access article distributed under the Creative Commons Attribution License, which permits unrestricted use, distribution, and reproduction in any medium, provided the original work is properly cited.

\begin{abstract}
The association of the cytotoxic T lymphocyte-associated antigen 4 (CTLA-4) gene and susceptibility to autoimmune thyroid diseases (AITDs) has been studied extensively. However, the results were not the same in different ethnic groups. We updated the meta-analysis of association of CTLA-4 gene polymorphisms with AITDs and summarized the results in specific ethnicity. The associations of A49G gene polymorphism with GD, A49G gene polymorphism with HT, CT60 gene polymorphism with GD, and CT60 gene polymorphism with HT were summarized based on the literatures published up to October 30, 2014, in English or Chinese languages. The participants involved in the studies of A49G with GD, A49G with HT, CT60 with GD, and CT60HT were 39004 subjects (in 51 studies), 13102 subjects (in 22 studies), 31446 subjects (in 22 studies), and 6948 subjects (in 8 studies), respectively. The pooled ORs of CTLA-4 gene polymorphisms with AITDs were larger than 1.00, and the 95\% CIs of ORs were statistically significant among whole population analyses. However, the subgroup analysis demonstrated that pooled ORs of A49G polymorphisms with GD among Africans or Americans are less than 1.00. The accumulated evidence suggests that the G allele mutant of A49G and CT60 increased the risks of HT and GD.
\end{abstract}

\section{Introduction}

Autoimmune thyroid diseases (AITDs) are the most popular autoimmune thyroid diseases; hyperthyroid Graves' disease (GD) and Hashimoto's (goitrous) thyroiditis (HT) are two common types of AITDs. It is well known that AITDs are caused partly by specific genetic background [1]. The association of the cytotoxic T lymphocyte-associated antigen 4 (CTLA-4) gene and susceptibility to AITDs has been studied extensively [2-4]. The CTLA-4 gene is located on the region of human chromosome $2 \mathrm{q} 33$ and encodes the immunoregulatory molecule. It is proved to be a key negative regulator of T-cell activity [5, 6]. Single nucleotide polymorphisms (SNPs) at position 49 in exon $1(+49 \mathrm{~A} / \mathrm{G}, \mathrm{A} 49 \mathrm{G}, \mathrm{rs} 231775)$ and +6230 G/A (CT60, rs3087243) showed an association with AITDs.
A comprehensive meta-analysis including 43 studies and more than 13,000 subjects was published in 2007 [7]. Subsequently, about 30 studies that investigated the relationship between the CTLA-4 gene SNPs and AITDs have been published. We designed the current systematic review and cumulative meta-analysis to include the most recent data and summarized the results with more genetic models.

\section{Methods}

2.1. Identification of Eligible Studies. The literature published up to October 30, 2014, in English or Chinese was searched in the MEDLINE, EMBASE, and China Biology Medicine disc (CBMdisc) databases. The search strategy was based on the key terms of "CTLA4," "CTLA-4," "cytotoxic T-cell 
TABLE 1: Characteristics of new studies included in the meta-analysis.

\begin{tabular}{|c|c|c|c|c|c|c|c|c|c|c|c|}
\hline \multirow{2}{*}{ Study } & \multirow{2}{*}{ Year } & \multirow{2}{*}{ Country } & \multirow{2}{*}{ Ethnicity } & \multirow{2}{*}{ Gene } & \multirow{2}{*}{ Disease } & \multicolumn{3}{|c|}{ Cases } & \multicolumn{3}{|c|}{ Controls } \\
\hline & & & & & & $\mathrm{AA}$ & $\mathrm{AG}$ & GG & $\mathrm{AA}$ & $\mathrm{AG}$ & GG \\
\hline Wang et al. [24] & 2001 & China & Asian & A49G & GD & 37 & 47 & 3 & 27 & 26 & 7 \\
\hline Zhou et al. [25] & 2003 & China & Asian & $\mathrm{A} 49 \mathrm{G}$ & GD & 32 & 14 & 4 & 5 & 5 & 10 \\
\hline Zhang et al. [8] & 2006 & China & Asian & A49G & GD & 37 & 18 & 1 & 26 & 25 & 29 \\
\hline Yao et al. [26] & 2006 & China & Asian & A49G & GD & 58 & 53 & 9 & 55 & 57 & 11 \\
\hline Yu et al. [32] & 2006 & China & Asian & A49G & GD & 51 & 36 & 13 & 26 & 46 & 28 \\
\hline Wang et al. [9] & 2007 & China & Asian & A49G & GD & 124 & 69 & 15 & 46 & 60 & 20 \\
\hline Yu et al. [27] & 2008 & China & Asian & A49G & GD & 67 & 45 & 13 & 13 & 27 & 29 \\
\hline Chong et al. [10] & 2008 & China & Asian & A49G & GD & 97 & 73 & 7 & 16 & 67 & 103 \\
\hline Cury et al. [11] & 2008 & Brazil & American & $\mathrm{A} 49 \mathrm{G}$ & GD & 15 & 58 & 43 & 6 & 64 & 47 \\
\hline Bicek et al. [12] & 2009 & Slovenia & Caucasian & A49G & GD & 17 & 73 & 33 & 14 & 52 & 24 \\
\hline Kimura et al. [13] & 2009 & Japan & Asian & A49G & GD & 210 & 143 & 62 & 10 & 42 & 32 \\
\hline Wang et al. [28] & 2010 & China & Asian & A49G & GD & 38 & 47 & 5 & 16 & 20 & 14 \\
\hline Guo et al. [29] & 2010 & China & Asian & A49G & GD & 26 & 52 & 24 & 12 & 47 & 41 \\
\hline Zhao et al. [14] & 2010 & China & Asian & A49G & GD & 1030 & 730 & 104 & 295 & 358 & 142 \\
\hline Pastuszak-Lewandoska et al. [15] & 2012 & Poland & Caucasian & A49G & GD & 7 & 6 & 1 & 97 & 77 & 18 \\
\hline Veeramuthumari et al. [16] & 2011 & India & Caucasian & A49G & GD & 32 & 37 & 11 & 71 & 56 & 24 \\
\hline Kimkong et al. [17] & 2011 & Thailand & Asian & A49G & GD & 61 & 49 & 22 & 54 & 73 & 26 \\
\hline Farra et al. [18] & 2012 & Lebanon & Caucasian & A49G & GD & 6 & 18 & 31 & 7 & 32 & 39 \\
\hline Pastuszak-Lewandoska et al. [19] & 2013 & Poland & Caucasian & A49G & GD & 12 & 9 & 3 & 945 & 823 & 156 \\
\hline Pastuszak-Lewandoska et al. [15] & 2012 & Poland & Caucasian & A49G & HT & 6 & 19 & 3 & 5 & 5 & 10 \\
\hline Zhou et al. [25] & 2003 & China & Asian & A49G & $\mathrm{HT}$ & 24 & 14 & 8 & 46 & 60 & 20 \\
\hline Yu et al. [27] & 2008 & China & Asian & A49G & $\mathrm{HT}$ & 41 & 34 & 5 & 15 & 64 & 22 \\
\hline Dallos et al. [20] & 2008 & Slovakia & Caucasian & A49G & $\mathrm{HT}$ & 13 & 34 & 16 & 13 & 27 & 29 \\
\hline Kucharska et al. [21] & 2009 & Poland & Caucasian & A49G & $\mathrm{HT}$ & 31 & 40 & 29 & 16 & 67 & 103 \\
\hline Bicek et al. [12] & 2009 & Slovenia & Caucasian & A49G & $\mathrm{HT}$ & 15 & 46 & 51 & 6 & 64 & 47 \\
\hline Sahin et al. [22] & 2009 & Turk & Caucasian & A49G & HT & 21 & 91 & 85 & 17 & 54 & 49 \\
\hline Farra et al. [18] & 2012 & Lebanon & Caucasian & A49G & HT & 6 & 31 & 36 & 16 & 20 & 14 \\
\hline Ying et al. [30] & 2012 & China & Asian & A49G & HT & 46 & 53 & 51 & 31 & 91 & 108 \\
\hline Pastuszak-Lewandoska et al. [19] & 2013 & Poland & Caucasian & A49G & HT & 14 & 8 & 3 & 7 & 48 & 43 \\
\hline Wang et al. [9] & 2007 & China & Asian & СT60 & GD & 138 & 46 & 5 & 30 & 61 & 26 \\
\hline Chong et al. [10] & 2008 & China & Asian & СT60 & GD & 125 & 48 & 4 & 735 & 516 & 84 \\
\hline Tsai et al. [23] & 2008 & China & Asian & СT60 & GD & 136 & 48 & 5 & 125 & 58 & 9 \\
\hline Bicek et al. [12] & 2009 & Slovenia & Caucasian & СТ60 & GD & 50 & 57 & 16 & 88 & 51 & 12 \\
\hline Kimura et al. [13] & 2009 & Japan & Asian & СT60 & GD & 267 & 127 & 21 & 82 & 59 & 12 \\
\hline Kimkong et al. [17] & 2011 & Thailand & Asian & CT60 & GD & 78 & 46 & 8 & 372 & 216 & 32 \\
\hline Qu et al. [31] & 2014 & China & Asian & CT60 & GD & 1989 & 487 & 114 & 1550 & 474 & 136 \\
\hline Dallos et al. [20] & 2008 & Slovakia & Caucasian & CT60 & HT & 31 & 28 & 4 & 20 & 50 & 25 \\
\hline Bicek et al. [12] & 2009 & Slovenia & Caucasian & СT60 & HT & 37 & 52 & 23 & 30 & 61 & 26 \\
\hline
\end{tabular}

lymphocyte associated antigen 4," “CD28," “CD152," “Graves' disease," "GD," "Hashimoto's thyroiditis," and "HT." Reference lists of relevant papers were reviewed to find additional studies. H.-F. Hou and X. Jin independently reviewed all studies and assessed the quality of each study according to the following inclusion criteria. (1) The publication was case-control study design, and the associations between A49G or CT60 genetic polymorphisms and AITDs were investigated. (2) Genotype distribution data were offered in both cases and controls. (3) For the overlapping data or the same papers, the largest population or the most recent study was included. (4) We limited the data to studies published in English and Chinese language. We compared our collection information with the data of Kavvoura et al. [7] on The Endocrine Society's Journals Online website (available at http://press.endocrine.org/journal/jcem) and adopted the unpublished studies provided in Kavvoura's meta-analysis.

2.2. Data Extraction. For published studies, two reviewers (H.-F. Hou and T. Sun) independently extracted data and 


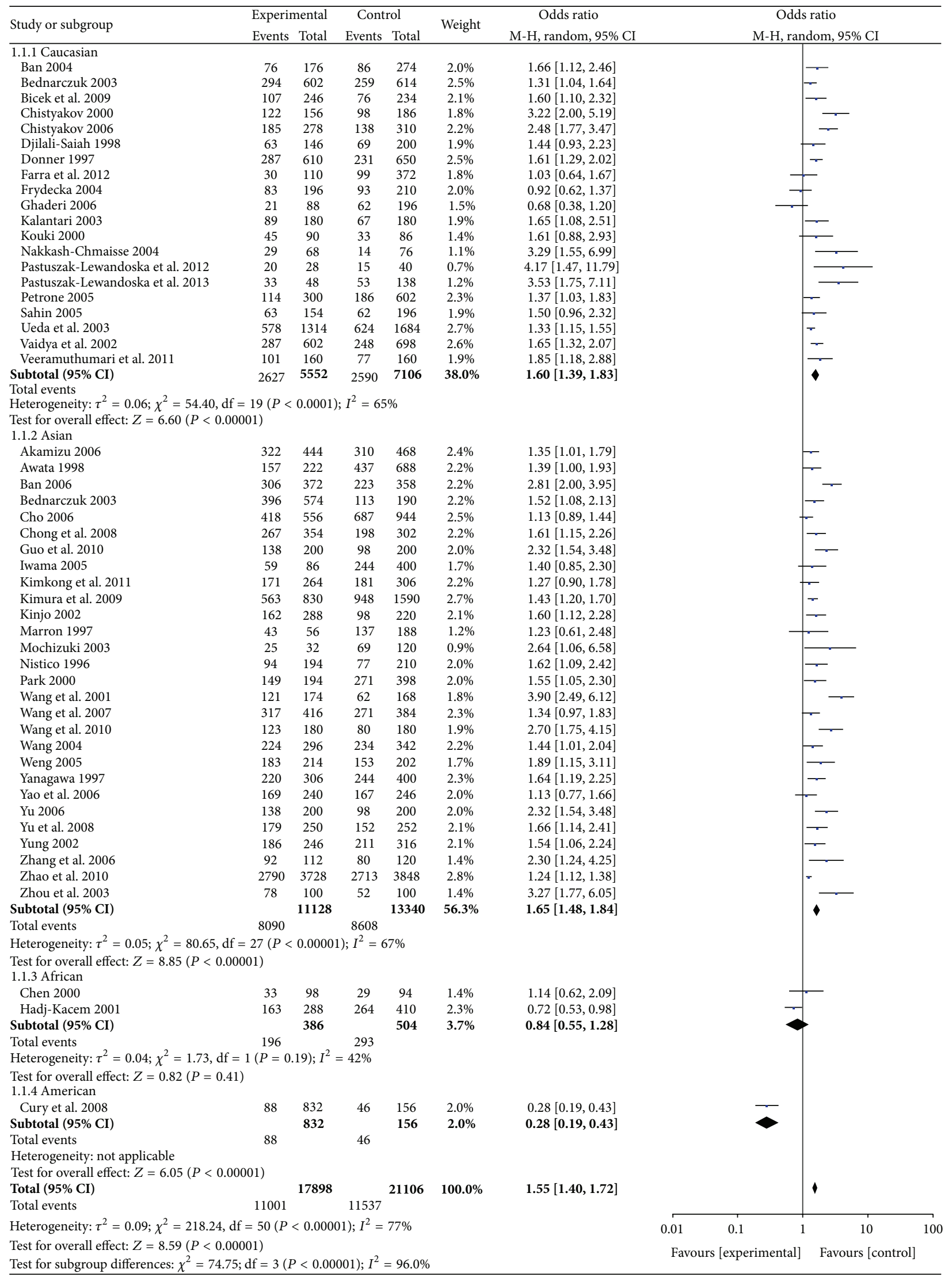

FIGURE 1: Forest plot of the association between an allele model of A49G polymorphism and GD. 


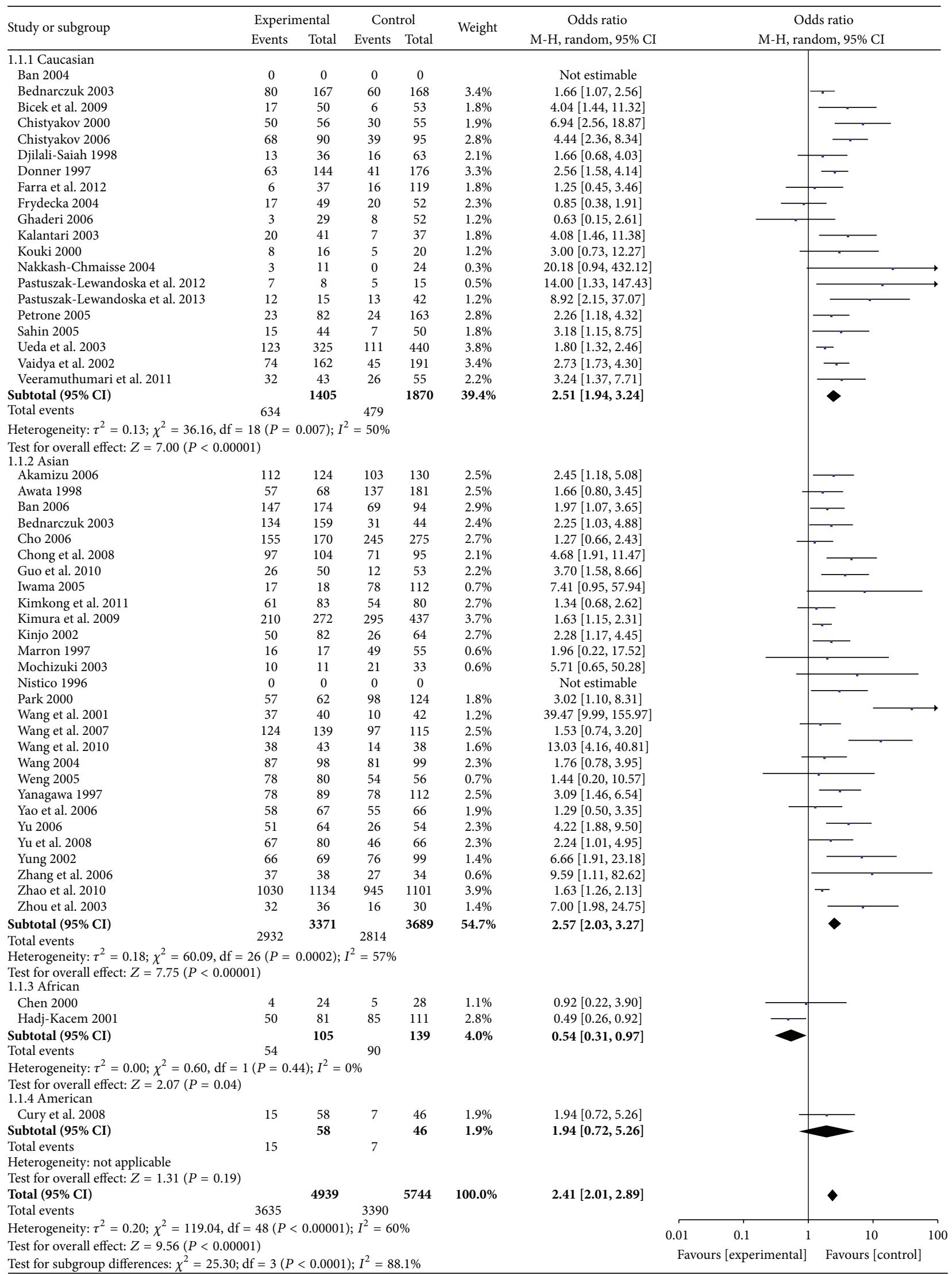

FIGURE 2: Forest plot of the association between an additive model of A49G polymorphism and GD. 


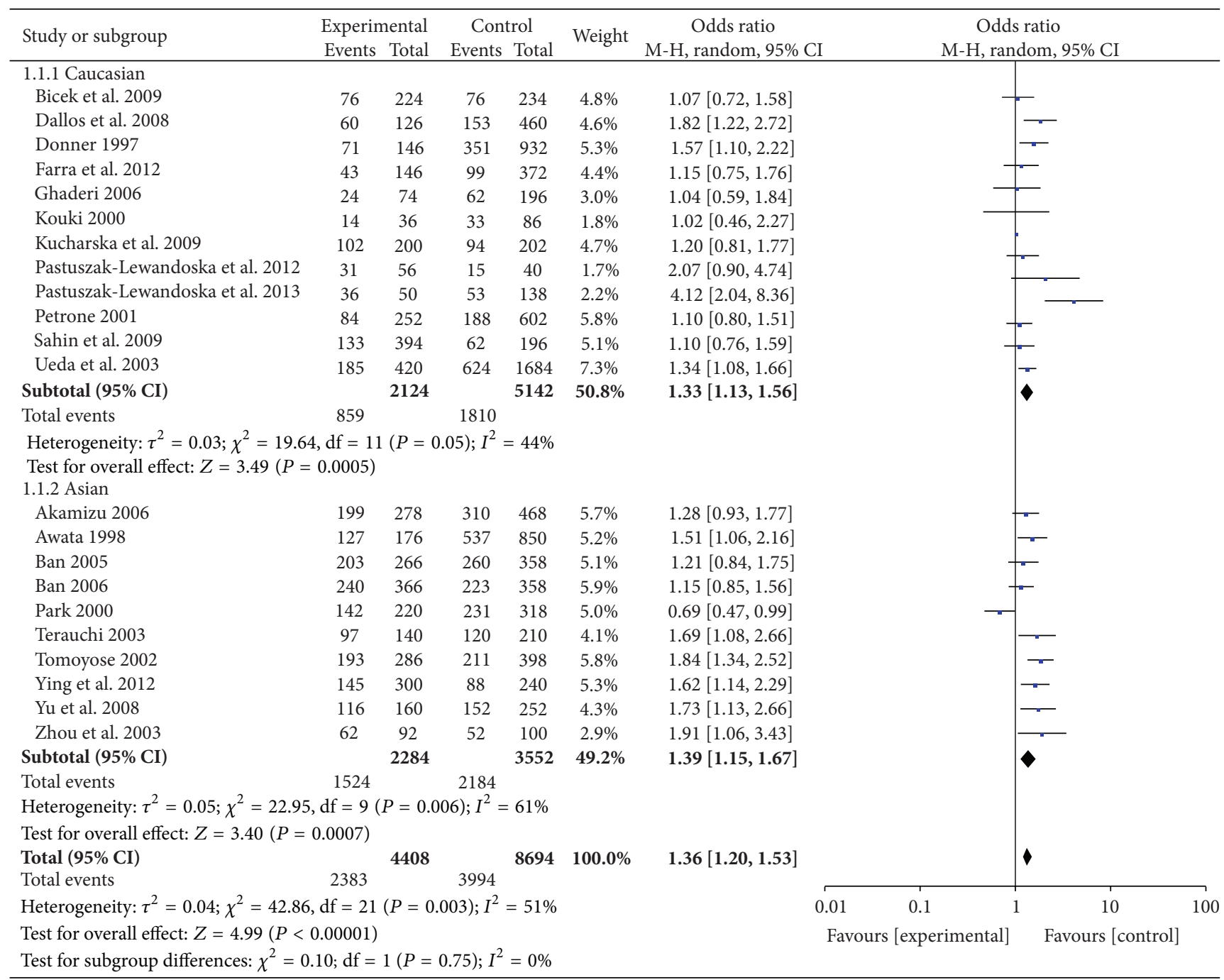

FIgURE 3: Forest plot of the association between an allele model of A49G polymorphism and HT.

resolved disagreements by discussion or with a third party ( $\mathrm{Li}$ QW) when necessary. We collected the following information carefully: author name, journal source, publication year, ethnicity of study population (Asian, Caucasian, African, and American), the number of individuals in case and control groups, and genotype distribution of cases and controls.

2.3. Meta-Analysis Methods. The analysis of data was performed with Review Manager 5.3 (The Cochrane Collaboration, Oxford, UK). Allele frequencies at the A49G or CT60 gene polymorphisms from the literatures were calculated by the allele counting method. Four genetic models, (1) allele contrast ( $G$ versus A), (2) additive genetic model (GG versus AA), (3) dominant model ( $G G+A G$ versus AA), and (4) recessive model ( $G G$ versus $A G+A A)$, were measured in this meta-analysis, and association values of the CTLA-4 genetic polymorphisms with risk of AITDs were estimated by odds ratios (ORs) and 95\% confidence intervals (CIs). We also assessed Hardy-Weinberg Equilibrium (HWE) of genotype frequencies in the control group with a chi-square test, and $P$ value $<0.05$ was considered to be significant. The heterogeneity across all studies was tested by the $I^{2}$ statistics and chi-square-based Q-test. The heterogeneity was considered to be significantly large when $P<0.10$ and $I^{2}>50 \%$. Then random effects model was used to combine eligible data. The statistical significance of pooled ORs was measured by the $Z$-test. Subgroup meta-analyses were conducted according to different ethnicities. In addition, sensitivity analysis was implemented to assess stability of the summary result by sequential removal of individual studies. Furthermore, publication bias was measured by funnel plots.

\section{Results}

3.1. Identification of Eligible Studies. Besides the 43 studies mentioned in Kavvoura et al's meta-analysis [7], 25 additional studies were included in this review (Table 1). Sixteen studies were English language publications [8-23] and 9 studies were published in Chinese [24-32]. Thus, the present updated meta-analysis consisted of 68 studies. 


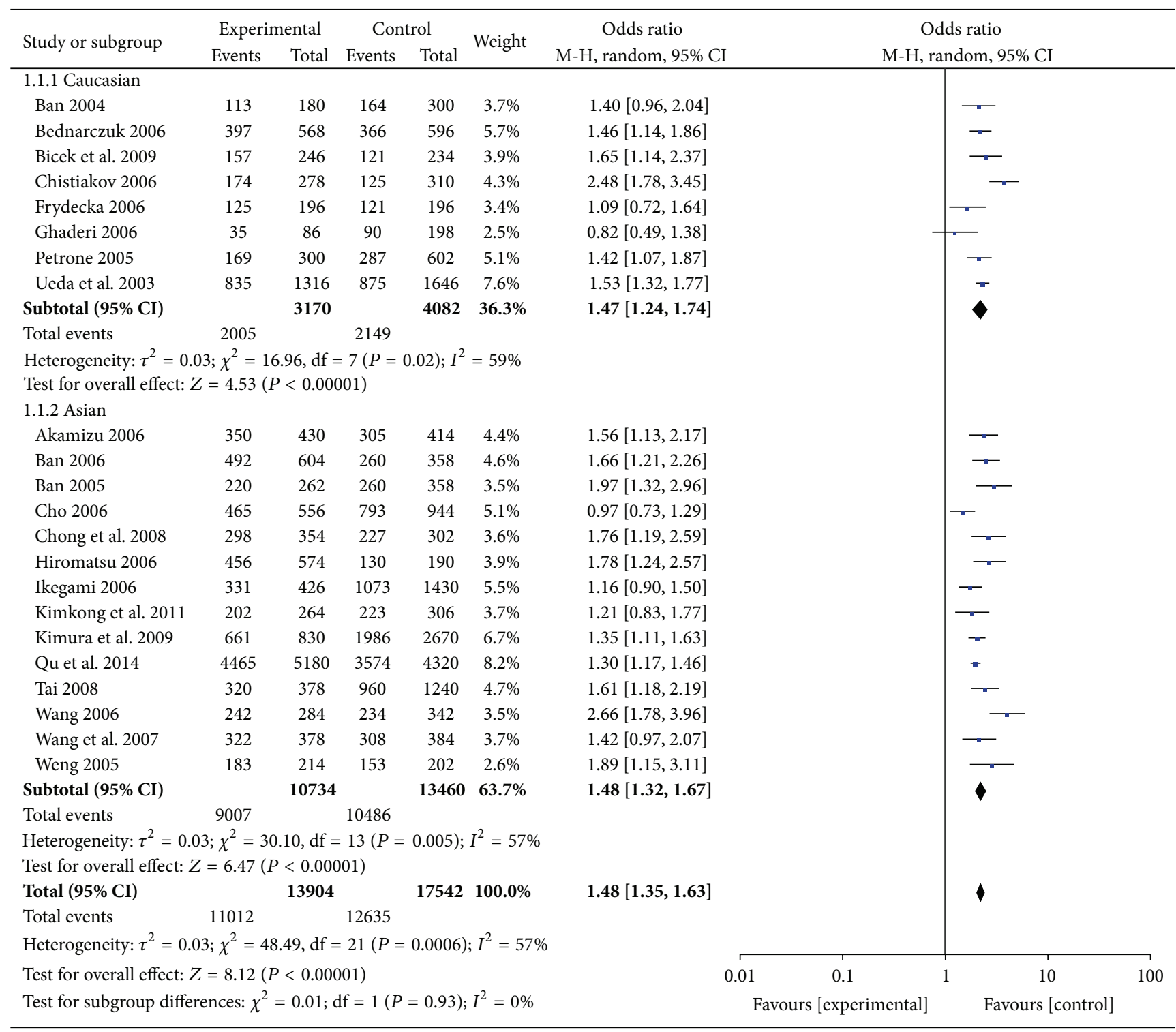

FIGURE 4: Forest plot of the association between an allele model of CT60 polymorphism and GD.

\subsection{Quantitative Analysis}

3.2.1. A49G Gene Polymorphism and GD. The summary OR of included studies was increased 1.55 -fold in susceptibility to GD in subjects with the G allele, and the associations of GD and A49G polymorphisms were statistically significant in an additive genetic model (GG versus $A A$ : $O R=2.41$, 95\% CI: 2.01-2.89), a dominant genetic model (GG + AG versus $\mathrm{AA}: \mathrm{OR}=1.76,95 \% \mathrm{CI}: 1.52-2.03)$, and a recessive genetic model (GG versus $\mathrm{AG}+\mathrm{AA}$ : $\mathrm{OR}=1.79,95 \% \mathrm{CI}$ : 1.58-2.02). The detailed results were shown in Figures 1 and 2 and Supplemental Figures 1 and 2 in Supplementary Material available online at http://dx.doi.org/10.1155/2015/747816.

The subgroup analysis was performed by ethnicity to decrease the heterogeneity. As shown in Figures 1 and 2, significant associations between A49G SNP and GD risk were identified in Asians and Caucasians.
3.2.2. A49G Gene Polymorphism and HT. The meta-analysis suggested (see Figure 3 and Supplemental Figures 3-5) that A49G polymorphisms increased the risk of HT significantly in the allele frequencies ( $\mathrm{G}$ versus $\mathrm{A}$ : $\mathrm{OR}=1.36,95 \% \mathrm{CI}: 1.20-$ 1.53 ), the additive genotype (GG versus $A A$ : $O R=2.10,95 \%$ CI: 1.75-2.51), the dominant genotype (GG + AG versus AA: $\mathrm{OR}=1.57,95 \% \mathrm{CI}: 1.26-1.96)$, and the recessive genotype (GG versus $\mathrm{AG}+\mathrm{AA}: \mathrm{OR}=1.46,95 \% \mathrm{CI}: 1.19-1.81)$. The subgroup analyses showed that A49G polymorphism was one of the risk factors for GD in Asians and Caucasians.

3.2.3. CT60 Gene Polymorphism and GD. The summary analyses of CT60 gene polymorphism and GD are shown in Figure 4 and Supplemental Figures 6-8. The pooled ORs of CT60 polymorphisms with GD in allele frequencies, the additive genetic model, the dominant genetic model, and the recessive genetic model were 1.48 (95\% CI: 1.35-1.63), 1.98 


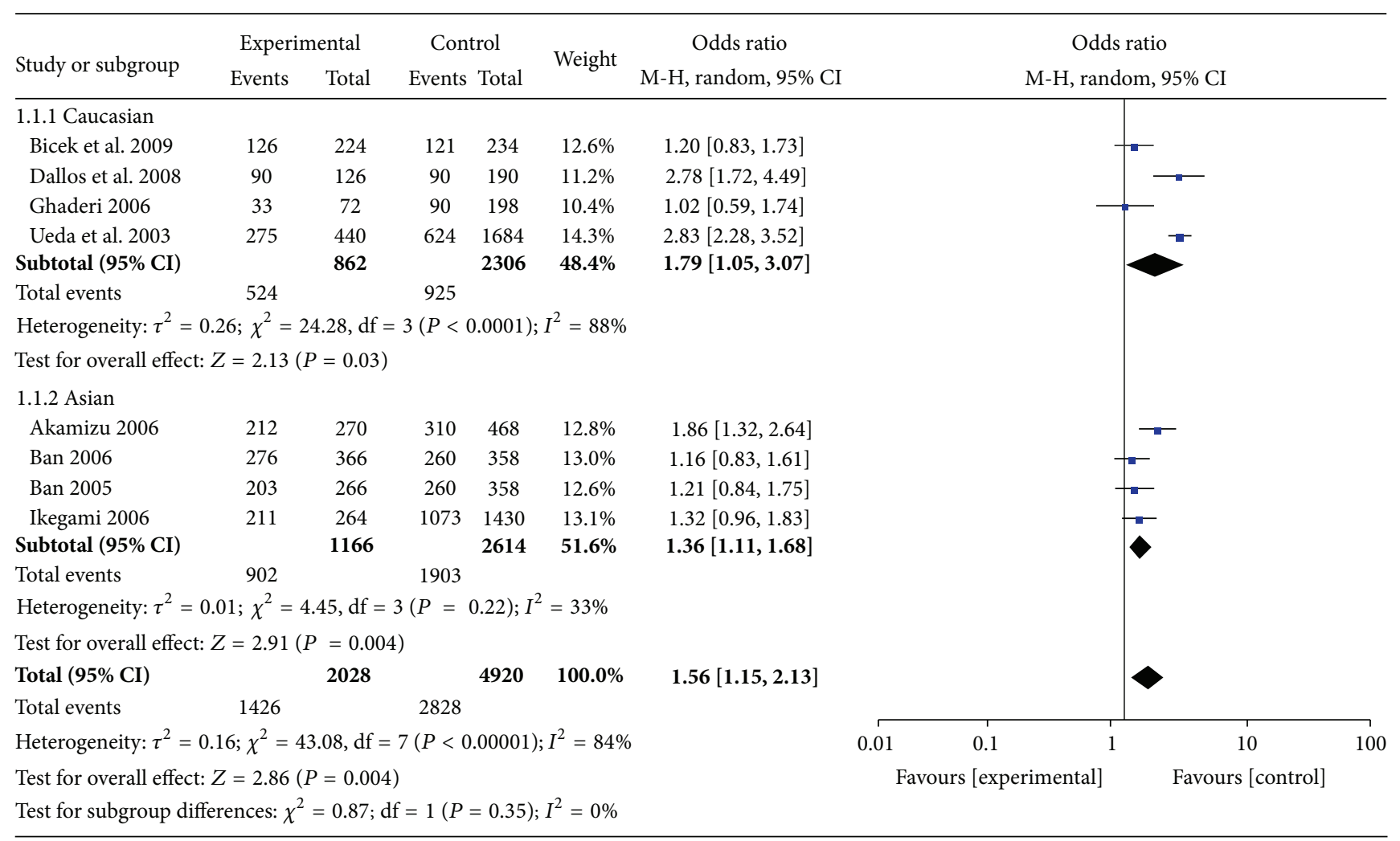

FIGURE 5: Forest plot of the association between an allele model of CT60 polymorphism and HT.

(95\% CI: 1.73-2.27), 1.72 (95\% CI: 1.52-1.96), and 1.56 (95\% CI: 1.39-1.76), respectively. The subgroup analyses suggested that CT60 polymorphism was a risk factor for GD in Asians and Caucasians.

3.2.4. CT60 Gene Polymorphism and HT. As shown in Figure 5 and Supplemental Figures 9-11, CT60 genetic polymorphisms increased HT risk significantly in the allele frequencies contrast ( $G$ versus A: OR $=1.56$, 95\% CI: $1.15-$ 2.13 ), the additive genetic contrast (GG versus $A A: O R=2.58$, 95\% CI: 1.33-5.01), the dominant genetic contrast (GG + AG versus $\mathrm{AA}: \mathrm{OR}=1.95,95 \% \mathrm{CI}: 1.20-3.15)$, and the recessive genetic contrast (GG versus $\mathrm{AG}+\mathrm{AA}$ : $\mathrm{OR}=1.79,95 \% \mathrm{CI}$ : 1.20-2.67). The subgroup analyses showed that CT60 genetic polymorphism was one of the risk factors for GD in Asians and Caucasians.

3.3. Publication Bias. In order to evaluate publication bias in this updated systematic review, Begg's Funnel plots were performed, and the results showed that no obvious asymmetry existed for the meta-analyses of A49G and CT60 genetic polymorphisms.

3.4. Sensitivity Analysis. In order to conduct sensitivity analyses, we calculated the pooled ORs through removing each study sequentially and leaving out certain studies, such as studies conducted among special population. The analyses showed that the results were not changed significantly. However, the summary results of the association between CT60 and HT among Caucasians were shifted in the sensitivity analyses.

\section{Discussion}

GD and HT are the most prevalent autoimmune thyroid diseases (AITDs), which represent two opposite pathogenic paths: hyperthyroidism in GD and thyroid destruction in HT $[15,19]$. Although the etiological mechanisms of GD and HT are not distinctly clarified, CTLA-4 gene polymorphisms (A49G and CT60) have been identified as the most important genetic factors in many genetic researches and genomewide association study (GWAS) $[2,12]$. A large-scale metaanalysis including 43 studies and more than 13,000 subjects was published in the present journal in 2007 [7]. The results identified the roles of A49G and CT60 gene polymorphism in AITDs. Subsequently, more than 30 studies repeatedly confirmed the associations of the CTLA-4 gene with GD and HT. The current updated meta-analysis included the most recent eligible studied and summarized the data in specific ethnicity.

A49G gene polymorphism was widely investigated for the susceptibility to AITDs; the G allelic gene variation was considered as a risk factor of GD and HT. Our current metaanalysis showed that A49G polymorphisms significantly increased the risk of GD in total population. Nevertheless, the 
genetic variation had a protective effect in Africans according to the additive model analysis. Furthermore, a total of 22 studies were summarized for the A49G gene polymorphism with HT. The results suggested that the polymorphism distinctly increases the risk of HT among Caucasians and Asians.

The G allele of CT60 gene is another focused genetic pathogenesis associated with $\mathrm{HT}$ and GD. A total of 22 studies were included in our meta-analysis for CT60 polymorphism and GD, and the pooled OR values indicated that $\mathrm{G}$ allele carriers might increase GD risk. Moreover, the summarized result involving 8 original studies suggested that CT60 polymorphisms were associated with susceptibility to HT among Caucasian and Asian population, except that no significant pooled OR was found in dominant genetic model of Caucasians.

In this updated meta-analysis, we guaranteed the stability of results with sensitivity analysis. No obvious publication bias existed according to funnel plot test. We performed heterogeneity test to assess the reliability of the results and conducted subgroup analysis.

There are some limitations in our study. The sample size in Africans or Americans was not large enough. More well-designed studies need to be conducted in Africans or Americans to clarify the associations of the CTLA-4 gene with AITDs.

\section{Conflict of Interests}

The authors declare that they have no competing interests.

\section{Authors' Contribution}

Hai-Feng Hou and Xu Jin contributed equally to this work.

\section{Acknowledgment}

This study was supported by the National Natural Science Foundation of China (Grant: NSFC, no. 81202170).

\section{References}

[1] B. Vaidya, P. Kendall-Taylor, and S. H. S. Pearce, "The genetics of autoimmune thyroid disease," The Journal of Clinical Endocrinology \& Metabolism, vol. 87, no. 12, pp. 5385-5397, 2002.

[2] H. Ueda, J. M. M. Howson, L. Esposito et al., "Association of the T-cell regulatory gene CTLA4 with susceptibility to autoimmune disease," Nature, vol. 423, no. 6939, pp. 506-511, 2003.

[3] K. J. Scalapino and D. I. Daikh, "CTLA-4: a key regulatory point in the control of autoimmune disease," Immunological Reviews, vol. 223, no. 1, pp. 143-155, 2008.

[4] J. Ni, L.-J. Qiu, M. Zhang et al., “CTLA-4 CT60 (rs3087243) polymorphism and autoimmune thyroid diseases susceptibility: a comprehensive meta-analysis," Endocrine Research, vol. 39, no. 4, pp. 180-188, 2014.

[5] P. Dariavach, M.-G. Mattei, P. Golstein, and M.-P. Lefranc, "Human Ig superfamily CTLA-4 gene: chromosomal localization and identity of protein sequence between murine and human CTLA-4 cytoplasmic domains," European Journal of Immunology, vol. 18, no. 12, pp. 1901-1905, 1988.

[6] L. Nisticò, R. Buzzetti, L. E. Pritchard et al., "The CTLA-4 gene region of chromosome $2 \mathrm{q} 33$ is linked to, and associated with, type 1 diabetes," Human Molecular Genetics, vol. 5, no. 7, pp. 1075-1080, 1996.

[7] F. K. Kavvoura, T. Akamizu, T. Awata et al., "Cytotoxic Tlymphocyte associated antigen 4 gene polymorphisms and autoimmune thyroid disease: a meta-analysis," Journal of Clinical Endocrinology and Metabolism, vol. 92, no. 8, pp. 3162-3170, 2007.

[8] Q. Zhang, Y.-M. Yang, and X.-Y. Lv, "Association of Graves' disease and Graves' ophthalmopathy with the polymorphisms in promoter and exon 1 of cytotoxic T lymphocyte associated antigen-4 gene.," Journal of Zhejiang University Science B, vol. 7, no. 11, pp. 887-891, 2006.

[9] P.-W. Wang, I.-Y. Chen, R.-T. Liu, C.-J. Hsieh, E. Hsi, and S.-H. H. Juo, "Cytotoxic T lymphocyte-associated molecule-4 gene polymorphism and hyperthyroid Graves' disease relapse after antithyroid drug withdrawal: a follow-up study," Journal of Clinical Endocrinology and Metabolism, vol. 92, no. 7, pp. 25132518, 2007.

[10] K. K. L. Chong, S. W. Y. Chiang, G. W. K. Wong et al., "Association of CTLA-4 and IL-13 gene polymorphisms with Graves' disease and ophthalmopathy in Chinese children," Investigative Ophthalmology and Visual Science, vol. 49, no. 6, pp. 2409-2415, 2008.

[11] A. N. Cury, C. A. Longui, C. Kochi et al., "Graves' disease in Brazilian children and adults: lack of genetic association with CTLA-4 +49A > G polymorphism," Hormone Research, vol. 70, no. 1, pp. 36-41, 2008.

[12] A. Bicek, K. Zaletel, S. Gaberscek et al., "49A/G and CT60 polymorphisms of the cytotoxic T-lymphocyte-associated antigen 4 gene associated with autoimmune thyroid disease," Human Immunology, vol. 70, no. 10, pp. 820-824, 2009.

[13] H. Kimura, Y. Kato, S. Shimizu, K. Takano, and K. Sato, "Association of polymorphism at position 49 in exon 1 of the cytotoxic T-lymphocyte-associated factor 4 gene with graves' disease refractory to medical treatment, but Not with amiodaroneassociated thyroid dysfunction," Thyroid, vol. 19, no. 9, pp. 975981, 2009.

[14] S.-X. Zhao, C.-M. Pan, H.-M. Cao et al., "Association of the CTLA4 Gene with Graves' disease in the chinese han population," PLoS ONE, vol. 5, no. 3, Article ID e9821, 2010.

[15] D. Pastuszak-Lewandoska, E. Sewerynek, D. Domańska, A. Gładyś, R. Skrzypczak, and E. Brzeziańska, "CTLA-4 gene polymorphisms and their influence on predisposition to autoimmune thyroid diseases (Graves' disease and Hashimoto's thyroiditis)," Archives of Medical Science, vol. 8, no. 3, pp. 415-421, 2012.

[16] P. Veeramuthumari, W. Isabel, and K. Kannan, "A study on the level of $\mathrm{T}(3), \mathrm{T}(4), \mathrm{TSH}$ and the association of $\mathrm{A} / \mathrm{G}$ polymorphism with CTLA-4 gene in graves' hyperthyroidism among south Indian population," Indian Journal of Clinical Biochemistry, vol. 26, no. 1, pp. 66-69, 2011.

[17] I. Kimkong, J. Nakkuntod, S. Sae-Ngow, T. Snabboon, Y. Avihingsanon, and N. Hirankarn, "Association between CTLA4 polymorphisms and the susceptibility to systemic lupus erythematosus and graves'disease in Thai population," Asian Pacific Journal of Allergy and Immunology, vol. 29, no. 3, pp. 229235, 2011. 
[18] C. Farra, J. Awwad, A. Fadlallah et al., "Genetics of autoimmune thyroid disease in the Lebanese population," Journal of Community Genetics, vol. 3, no. 4, pp. 259-264, 2012.

[19] D. Pastuszak-Lewandoska, D. Domańska, M. Rudzińska et al., "CTLA-4 polymorphisms (+49 A/G and $-318 \mathrm{C} / \mathrm{T})$ are important genetic determinants of AITD susceptibility and predisposition to high levels of thyroid autoantibodies in Polish children-Preliminary study," Acta Biochimica Polonica, vol. 60, no. 4, pp. 641-646, 2013.

[20] T. Dallos, M. Avbelj, E. Barák et al., "CTLA-4 gene polymorphisms predispose to autoimmune endocrinopathies but not to celiac disease," Neuroendocrinology Letters, vol. 29, no. 3, pp. 334-340, 2008.

[21] A. M. Kucharska, E. Gorska, M. Wasik, B. Pyrzak, and U. Demkow, "Expression of CD152 (CTLA-4) in children with autoimmune thyroiditis and $+49 \mathrm{~A} / \mathrm{G}$ polymorphism of exon 1 of the CTLA-4 gene," Journal of Physiology and Pharmacology, vol. 60, pp. 77-80, 2009.

[22] M. Sahin, A. Gursoy, and M. F. Erdogan, "Cytotoxic T lymphocyte-associated molecule- 4 polymorphism in Turkish patients with Hashimoto thyroiditis," International Journal of Immunogenetics, vol. 36, no. 2, pp. 103-106, 2009.

[23] S.-T. Tsai, C.-Y. Huang, F.-S. Lo et al., "Association of CT60 polymorphism of the CTLA4 gene with Graves' disease in Taiwanese children," Journal of Pediatric Endocrinology and Metabolism, vol. 21, no. 7, pp. 665-672, 2008.

[24] L. Wang, H. Yu, and S. H. Yan, "The association of cytotoxic T lymphocyte-associated antigen 4 gene polymorphism with type 1 diabetes mellitus and autoimmune thyroid diseases in Chinese Han population," Chinese Journal of Endocrinology and Metabolism, vol. 17, pp. 228-231, 2001.

[25] W. X. Zhou, B. Y. Shi, H. F. Wang, M. Q. Hou, X. Y. Wu, and W. Cui, "The correlation between the cytotoxic T lymphocyte associated antigen 4 gene polymorphism and autoimmune thyroid disease," Journal of Xian Jiaotong University (Medical Sciences), vol. 24, no. 2, pp. 170-173, 2003.

[26] B. Yao, L. M. Hao, J. H. Yan, J. P. Weng, and Y. B. Li, "Association between the CTLA-4 gene polymorphism and Graves'disease in the Southern Chinese Han population," Chinese Journal of Endocrinology and Metabolism, vol. 22, no. 4, pp. 363-364, 2006.

[27] Z. Y. Yu, J. A. Zhang, H. B. Maier et al., "Association of polymorphism of protein tyrosine phosphatase nonreceptor22 gene with AITD," Chinese Journal of Cellular and Molecular Immunology, vol. 24, no. 8, pp. 804-807, 2008.

[28] S. H. Wang, J. Gao, and H. Zhang, "Association of CTLA-4 gene exon 1 polymorphism with graves disease in han population," Journal of Qinghai Medical College, vol. 31, pp. 225-245, 2010.

[29] Z. Guo, X. Chen, P. Wu, and G. Wu, "Relationships between CTLA-4 gene polymorphism and Graves'disease in Han population in western region of Guangdong province," Journal of Guangdong Medical College, vol. 28, pp. 1-3, 2010.

[30] J. Ying, H. Shao, R. Pan, P. Li, H. Zhang, and X. Zen, "Study on the correlation between CTLA-4, PTPN22 gene polymorphism and Hashimoto's thyroiditis in Wenzhou population," Journal of Wenzhou Medical College, vol. 42, pp. 346-353, 2012.

[31] R. Qu, S. Li, J. Zheng, W. Liu, B. Liu, and Z. Song, "Association between CTLA4 gene polymorphism and the susceptibility to Graves' disease," Chinese Journal of Endocrinology and Metabolism, vol. 30, pp. 35-37, 2014.

[32] Q. Yu, D. Chen, Z. Xiao, and Y. Wang, "Association of polymorphism of CTLA-4 gene exon 1 with Graves disease in Cantonese Han population," Anatomy Research, vol. 28, pp. 278-280, 2006. 


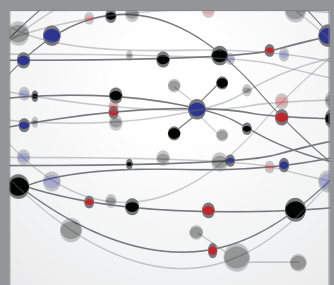

The Scientific World Journal
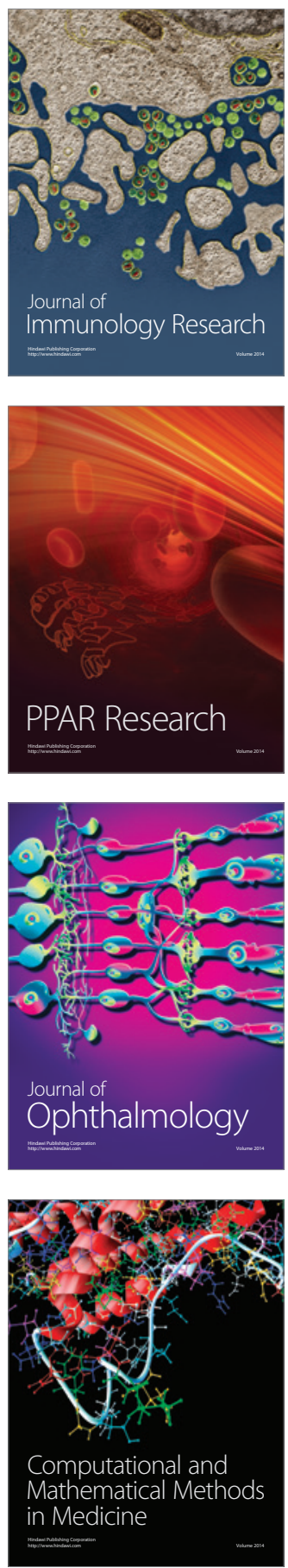

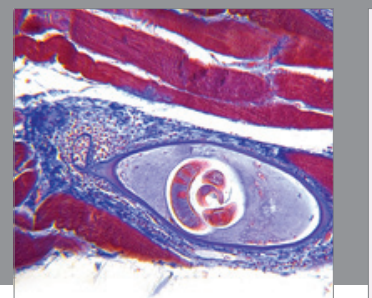

Gastroenterology

Research and Practice
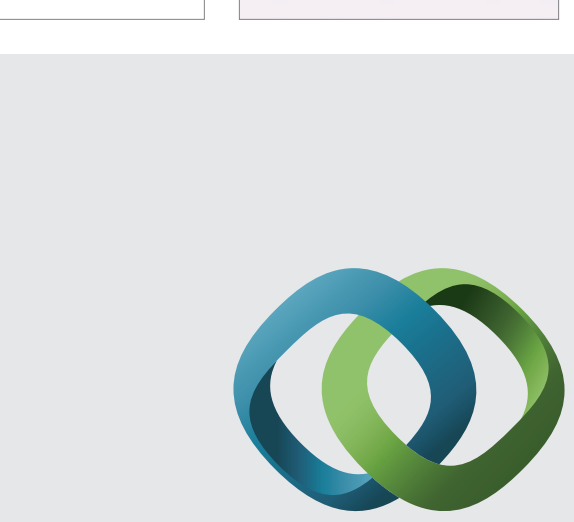

\section{Hindawi}

Submit your manuscripts at

http://www.hindawi.com
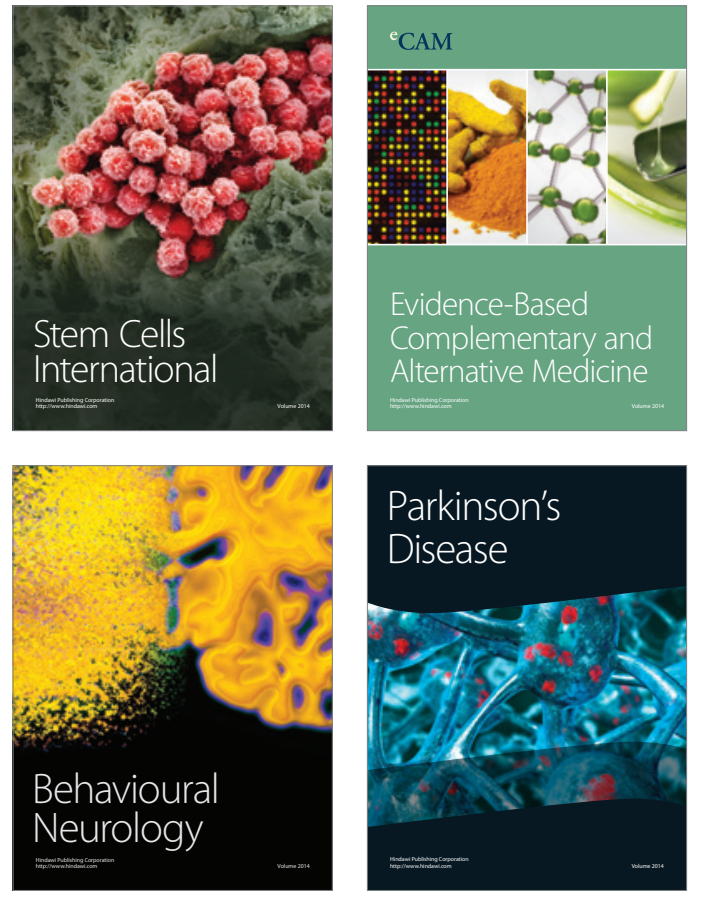
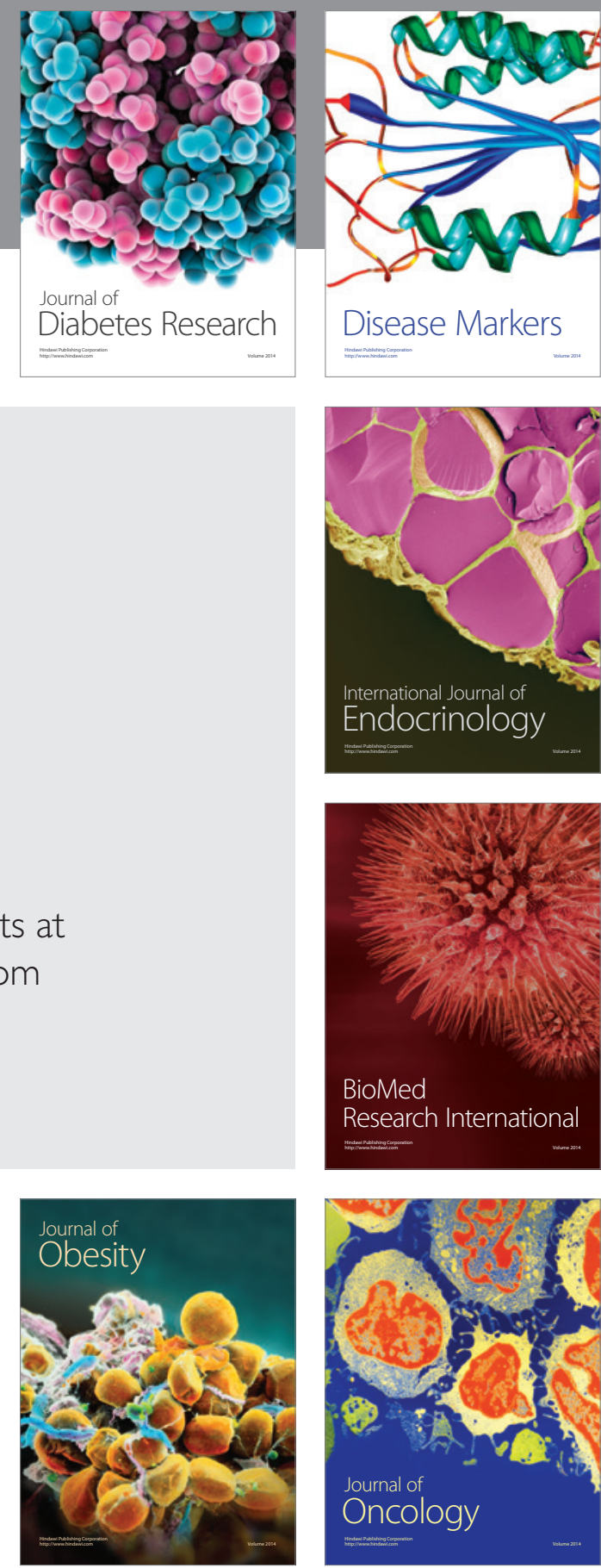

Disease Markers
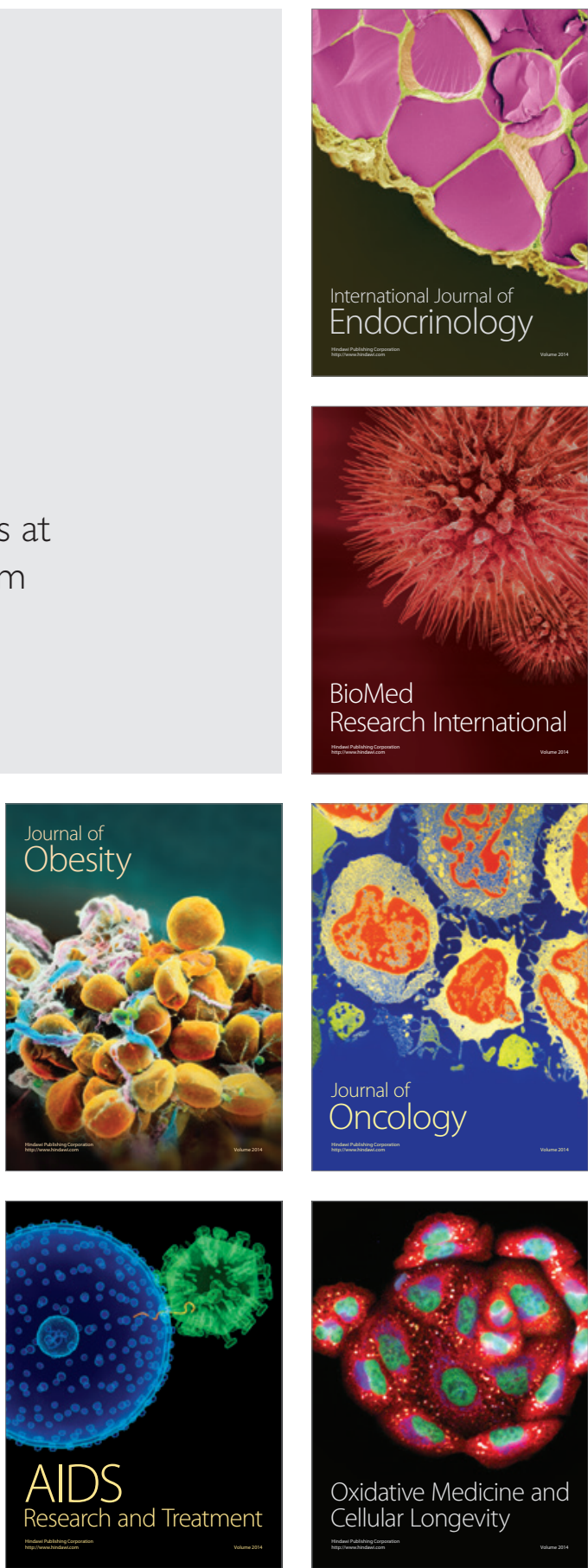